\title{
Center for Multiscale Plasma Dynamics: Report on Activities (UCLA/MIT), 2009-2010.
}

The final "phaseout" year of the CMPD ended July 2010; a no cost extension was requested until May 2011 in order to enable the MIT subcontract funds to be fully utilized. Research progress over this time included verification and validation activities for the BOUT and BOUT++ code, studies of spontaneous reconnection in the VTF facility at MIT, and studies of the interaction between Alfvén waves and drift waves in LAPD. The CMPD also hosted the 6th plasma physics winter school in 2010 (jointly with the NSF frontier center the Center for Magnetic Self-Organization, significant funding came from NSF for this most recent iteration of the Winter School).

\section{Winter School 2010}

\section{Shear Flows and Momentum Transport in Lab and Astrophysical Plasmas}

\author{
Date January 4th - 8th, 2010 \\ Location. UCLA Department of Physics and Astronomy
}

The Center for Multiscale Plasma Dynamics and The Center for Magnetic Self-Organization sponsored a winter school on "Shear Flows and Momentum Transport in Lab and Astrophysical Plasmas" at UCLA on January 4th - 8th, 2010. The school consisted of six days of coordinated lectures on the basic physics of sheared flow and momentum transport in plasmas. Attended by over 100 graduate students and postdocs, lectures were given by leaders in the field of momentum transport in plasmas and neutral fluids on a variety of topics, both experimental and theoretical. Winter School students were provided with lecture notes and problems to aid in their understanding. Copies of the lectures can be downloaded at http://cmpd.pna.ucla.edu/2010/ - the full schedule is hyper-linked to lecture notes.

\section{Research}

We are happy to report progress on many fronts.

\section{- Verification and Validation of BOUT and BOUT++}

Over 2009-2010, significant effort was made in applying the 3D Braginskii turbulence code BOUT (and the re-write of this code, BOUT++) to simulation of plasmas in the Large Plasma Device. The code was originally written to simulate tokamak edge plasmas and has flexible specification of magnetic geometry. The code was successfully modified to simulate cylindrical LAPD plasmas and was verified against linear calculations for instabilities (resistive drift waves, Kelvin-Helmholtz and rotation interchange). Full nonlinear simulations were performed, using measured LAPD profiles (initial simulations have been electrostatic and have ignored gradients in temperature and temperature fluctuations for simplicity). Comparisons of these simulations with measurements in LAPD plasmas reveal very good qualitative and semi-quantitative agreement, in particular in frequency spectrum, spatial correlation and amplitude probability distribution function of density fluctuations. Three papers have been published as a result of this work, and an invited talk was given at the 2010 APS Division of Plasma Physics meeting by LLNL collaborator Maxim Umansky. 
Personnel involved in this effort: Pavel Popovich (UCLA Postdoc), Troy Carter, Maxim Umansky (LLNL), Brett Friedman (UCLA Graduate Student), David Schaffner (UCLA Graduate Student)

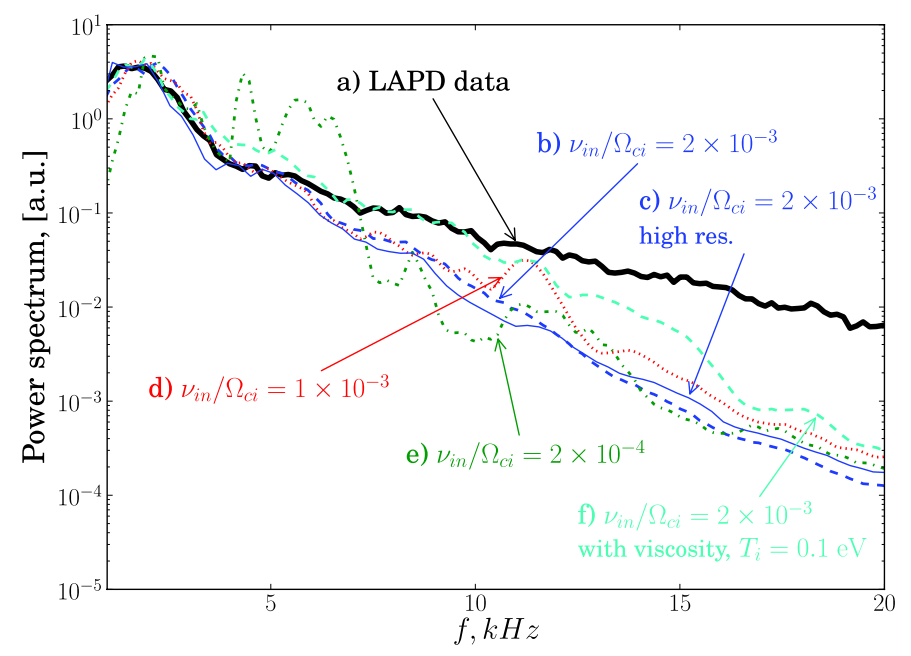

Figure 1: Comparison of fluctuation power spectrum from LAPD data and BOUT simulation.

\section{- Studies of nonlinear Alfvén waves in LAPD}

Studies of nonlinear processes associated with Alfvén waves have continued, in particular focusing on beat-wave interactions. A new technique for manipulation and control of gradient-driven instabilities through nonlinear interaction with Alfvén waves has been developed. A narrow, field-aligned density depletion is created in LAPD, resulting in coherent, unstable fluctuations on the periphery of the depletion. Two independent shear Alfvén waves are launched along the depletion at separate frequencies, creating a nonlinear beat-wave response at or near the frequency of the original instability. When tuned to the instability frequency, the beat-wave can resonantly drive the unstable mode. The beating waves can also drive nearby damped modes when the frequency is detuned from the instability frequency. When the beat-wave-driven damped mode has sufficient amplitude, the original unstable mode is suppressed, leaving only the beat-wave response, generally at lower amplitude. An invited presentation was given at the 2010 APS DPP meeting by UCLA graduate student David Auerbach.

Personnel involved: Troy Carter, Dave Auerbach (UCLA graduate student), Pavel Popovich (UCLA postdoc).

\section{- Studies of spontaneous reconnection in VTF (MIT subcontract)}

Work on magnetic reconnection, focused on the "trigger" mechanism for fast reconnection, continued at MIT on the VTF facility. The trigger for fast reconnection in toroidal experiments was studied using a magnetic x-type geometry in the strong guide-field regime. The onset of reconnection was found to occur asymmetrically: the reconnection begins on one side of the torus and propagates around approximately at the Alfvén speed. The fast reconnection occurs only in the presence of a global plasma mode, which breaks the axisymmetry and enables the current at the $x$-line to decrease sharply. A simple semi-empirical model was developed and used to describe the onset's growth rate. An invited talk was given on this topic at the 2010 APS DPP meeting by Noam Katz.

Personnel involved: Jan Egedal, Noam Katz (MIT graduate student) 


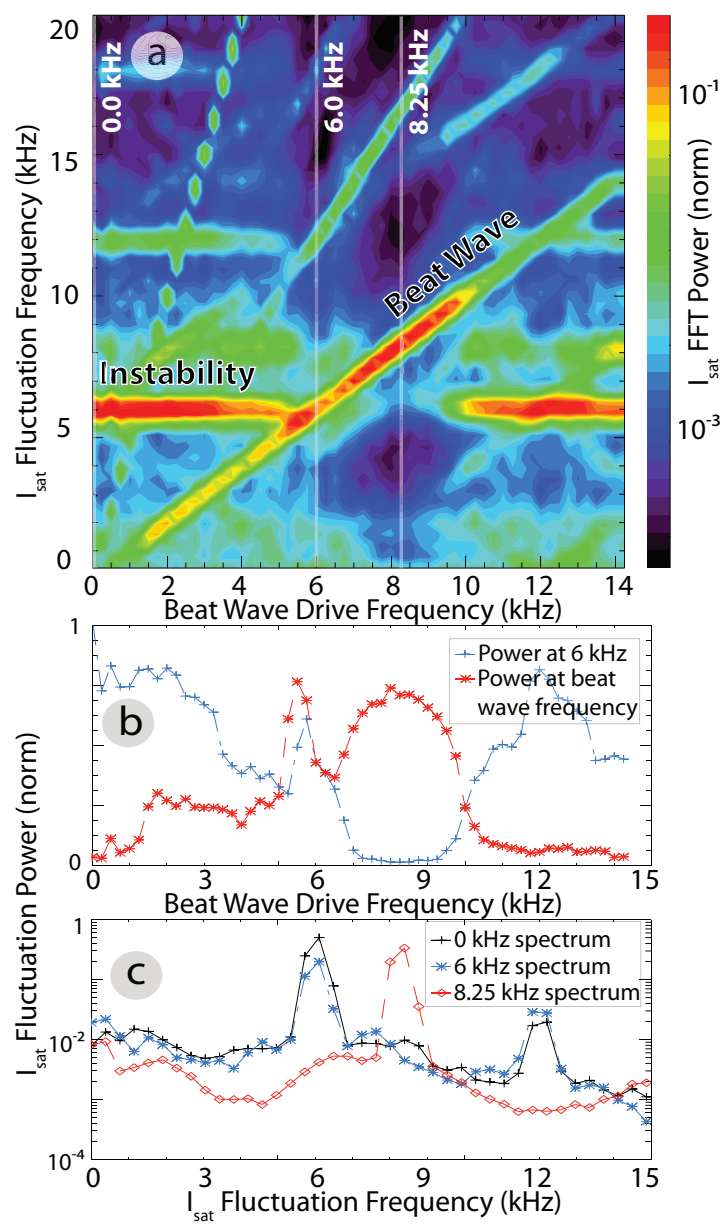

Figure 2: Demonstration of resonant drive and suppression of gradient driven instabilities in LAPD. (a) Measured $I_{\text {sat }}$ fluctuation power as a function of the shear Alfvén wave beat frequency (x axis) and FFT frequency (y axis). The beat frequency is varied shot-to-shot. (b) Power at the spontaneous mode frequency $(f=6 \pm 0.4 \mathrm{kHz})$ and at the SAW beat frequency $\left(f=f_{\text {beat }} \pm 0.4 \mathrm{kHz}\right)$. (c) $I_{\text {sat }}$ fluctuation power spectra at $0.0,6.0$, and $8.25 \mathrm{kHz}$ SAW beat frequency (marked with vertical lines on the 2-D multi-spectrum plot above in part a). 


\section{Personnel}

We list people involved in center activities, some are supported by the center. All participate in meetings.

\subsection{Senior Scientists}

UCLA: Professor Troy Carter (Director), Professor Walter Gekelman, Dr. Tony Peebles, Dr. Lothar Schmitz, Dr. Jim Maggs, Dr. Pat Pribyl and Dr. Steven Vincena. MIT: Professor Jan Egedal

\subsection{Post-docs}

Pavel Popovich, theoretical/numerical post-doc trained in Kurchatov and Lausanne.

\subsection{Graduate Students}

Brett Friedman, theoretical graduate student at UCLA, supported by DOE Fusion Energy Sciences Graduate fellowship. Jon Hillesheim (DOE Fusion Fellow), David Schaffner, and David Auerbach, experimental students at UCLA. Noam Katz, experimental student at MIT. 


\section{References}

[1] P. Popovich, M.V. Umansky, T.A. Carter, and B. Friedman, "Analysis of plasma instabilities and verification of the BOUT code for the Large Plasma Device," Physics of Plasmas 17, 2010, 102107, 11 pages.

[2] P. Popovich, M.V. Umansky, T.A. Carter, and B. Friedman, "Modeling of plasma turbulence and transport in the Large Plasma Device," Physics of Plasmas 17, 2010, 122312.

[3] M.V. Umansky, P. Popovich, T.A. Carter, B. Friedman, and W. Nevins, "Numerical simulation and analysis of plasma turbulence in LAPD," Phys. Plasmas, accepted, to appear May 2011.

[4] D. W. Auerbach, T. A. Carter, S. Vincena, and P. Popovich, "Control of gradient-driven instabilities through shear Alfvén beat waves," Physical Review Letters 105, 2010, 135005, 4 pages.

[5] D. W. Auerbach, T. A. Carter, S. Vincena, and P. Popovich, "Resonant drive and nonlinear suppression of gradient-driven instabilities via interaction with shear Alfvén waves," Phys. Plasmas, accepted, to appear May 2011.

[6] N. Katz, J. Egedal, W. Fox, A. Le, J. Bonde, and A. Vrublevskis, "Laboratory Observation of Localized Onset of Magnetic Reconnection", (2010) Phys. Rev. Lett. 104, 255004.

[7] Noam Katz, Jan Egedal, William (Will) Fox, Ari Le, Arturs Vrublevskis and Jeff Bonde, "Experimental Investigation of the Trigger Problem in Magnetic Reconnection," Phys. Plasmas, accepted, to appear May 2011.

[8] S. Zhou, W.W. Heidbrink, H. Boehmer, R. McWilliams, T.A. Carter, S. Vincena, S. Tripathi, P. Popovich, B. Friedman and F. Jenko, "Turbulent Transport of Fast Ions in the Large Plasma Device," Physics of Plasmas 17, 2010, 092103, 10 pages.

\section{Colloquia, Outreach, Invited Talks at Large meetings, etc.}

Troy Carter's talks etc. 2009-2010 seminars not shown.

1. (Aug 2009) "Interaction between Alfvén waves and drift-Alfvén waves in a laboratory plasma," International Symposium on Cutting Edge Plasma Physics, ICTP, Trieste, Italy

2. (Nov 2009) "Modification of turbulence and turbulent transport associated with a confinement transition in LAPD," 51st Annual Meeting of the APS Division of Plasma Physics, Atlanta, GA

3. (Feb 2010) "Control of gradient-driven instabilities using shear Alfvén beat waves," Eighth International Workshop on Nonlinear Waves and Turbulence in Plasmas, La Jolla, CA

4. (Mar 2010) "Overview of research using the Basic Plasma Science Facility at UCLA," Workshop on Labortory Basic Plasma Research, Berlin, Germany.

5. (Jun 2010) "Studies of instabilities, turbulence and transport in the Large Plasma Device," Workshop on Electric Fields, Turbulence and Self-Organisation in Magnetised Plasmas, Dublin, Ireland.

6. (Jul 2010) "Studies of waves, turbulence and transport in the Large Plasma Device," Workshop of Kinetic-Scale Turbulence in Laboratory and Space Plasmas, Isaac Newton Institute, Cambridge University, Cambridge, UK.

7. (Aug 2010) "Control of gradient-driven instabilities through nonlinear interaction with shear Alfvén waves," International Congress on Plasma Physics, Santiago, Chile. 
Pavel Popovich: 2010 Transport Task Force meeting, Simulation of Turbulence and Transport in LAPD Maxim Umansky: 2010 APS DPP meeting, invited talk, Towards Validation of Turbulence Simulation Using LAPD Data

Noam Katz: 2010 APS DPP meeting, invited talk, Experimental Investigation of the Trigger Problem in Magnetic Reconnection 